\title{
National Health Education, Medical Education, and Healthcare Legal System in China
} \author{
Meng Qingyue ${ }^{1,2}$, Wang Longde ${ }^{4}$ \\ 1. China Center for Health Development Studies, Peking University, Beijing 100191, China \\ 2. School of Public Health, Peking University, Beijing 100191, China \\ 3. Institute of Medical Education, Peking University, Beijing 100191, China \\ 4. Chinese Preventive Medicine Association, Beijing 100021, China
}

Liu Xiaoyun ${ }^{1}$, Chang Chun ${ }^{2}$, Hou Jianlin ${ }^{3}$, Yang Jian ${ }^{2}$, Tian Chuansheng ${ }^{4}$, Ji Ying ${ }^{2}$, Hu Dan ${ }^{1}$,

\begin{abstract}
Although China has achieved great success in the reform of its medical system, it still faces considerable challenges in national health education, medical and healthcare talent cultivation, and healthcare legal system development. This paper summarizes local and international experiences, analyzes the current situation and challenges, and recommends policies to improve China's national health education, medical and healthcare education, and healthcare legal system. These policy recommendations include: developing a compulsory national health education system, aligning medical education for healthcare talents with social needs, strengthening the development of general practitioners, improving the service level and accessibility of primary medical care, and promoting the incorporation of "Parent Law" into medicine and health. This paper also examines and analyzes the existing medical and healthcare laws and regulations.
\end{abstract}

Keywords: national health; health education; medical education; healthcare laws

\section{Introduction}

Since the new phase of health system reform in 2009, China has achieved great success in expanding its coverage of financial protection mechanisms, providing essential public health programs to all citizens, strengthening the capacity of primary healthcare institutions, and establishing a national essential medicine system. These accomplishments have laid a solid foundation for further reform for attaining the overall goal of instituting and improving the basic healthcare system to cover both urban and rural residents. At the same time, great challenges are still being faced due to the complex and systematic nature of the health system reform. National health education system lags behind, and new healthcare talent needs to be developed based on population's healthcare needs. Additionally, China's healthcare legal system and multi-sectoral cooperation need to be strength- ened. Therefore, it requires continuous improvement of reform policies and effective implementation of solutions in response to newly emerging issues.

This study aimed to analyze local and international experiences and explore new models of national health education, medical education, and healthcare legal system that align with the overall goal of universal health coverage. The main objectives of this research were to (1) design a "compulsory health education system" for children and adults to improve their health literacy for disease prevention and control, which could further improve national health; (2) propose a new system for professional health education and training with the characteristics of "integrative medicine" to repair the discrepancy between educational investments and labor market demands and improve the efficiency of human resource allocation; and (3) study the enforced legal and regulatory system of the "Development Strategy for National

Received date: December 15, 2016; Revised date: March 20, 2017

Corresponding author: Wang Longde, Chinese Preventive Medicine Association, President; Chinese Academy of Engineering, Academician. Major research fields include epidemiology and public health. E-mail: wangld@nhfpc.gov.cn

Funding program: CAE Advisory Project "Development Strategy for National Health Promotion and Medical and Healthcare Undertakings in China" (2014-ZD-06) Chinese version: Strategic Study of CAE 2017, 19 (2): 050-054

Cited item: Liu Xiaoyun et al. National Health Education, Medical Education and Healthcare Legal System in China. Strategic Study of CAE, https://doi.org/10.15302/ J-SSCAE-2017.02.008 
Health Promotion and Medical and Health Undertakings in China," and generate recommendations to improve the structure and nature of the system, as well as the benefits of implementing laws and regulations.

\section{Current situation of national health education, medical education, and healthcare legal system}

\subsection{Current status of national health education}

The Ottawa Charter for Health Promotion proposes five key action areas to promote health: building healthy public policy, creating supportive environments, strengthening community action, developing personal skills, and re-orienting healthcare services toward prevention of illness and promotion of health. This charter states that health education should be implemented in schools and communities as well as workplaces, combined with policy and environmental support. The 8th Global Conference on Health Promotion in Helsinki proposed the theory of "Health in All Policies" (HiAP). The premise of the theory is that good health enhances the quality of life, increases capacity for learning, strengthens families and communities, and improves workforce productivity. However, various healthcare issues and inequalities are rooted in social and economic problems beyond the health sector and policy domains. Policies implemented in all sectors can have a profound effect on the population's health and health equity. HiAP is an approach that acknowledges the social determinants of health to improve the population's health and health equity.

\subsubsection{Development of China's health education system}

China started developing a health education system centered on health administrative departments and institutions ever since the founding of new China. At present, China's health education system includes four levels, from the country, provincial, and municipal, to the district/county level. Health administrative departments and professional health education institutions constitute the main body of the system, with communities, schools, workplaces, hospitals, enterprises, media, and other social forces involved in it.

\subsubsection{Health education funding}

Health education is one of the basic public health services. Presently, health education funding resources in China mainly come from basic public health service equalization programs and health literacy promotion projects. China launched the basic public health service equalization program in 2009. Central and local governments have allocated funds for this program in different proportions. The government health expenditure per capita for the program increased from 15 yuan in 2009 to 45 yuan in 2016. In 2012, the Chinese government started implementing the current national health literacy promotion project subsidized by the central government. The project funding was 0.238 billion yuan in 2012 and increased to 0.259 billion yuan in 2014 [1]

2.1.3 Implementation of health education policies and activities Several national policy documents related to health education have been enacted in China. Under the guidance of these policies, ever since the 1950s, especially from the mid-1980s till the present, China has conducted health education activities led by professional health education institutions and administrative departments (e.g., free clinics on World Health Day and dissemination of health education promotional materials), program-based health education and promotional activities (e.g., development of "Health-Promoting Schools" in collaboration with WHO), and multi-sectoral collaborative health programs (e.g., "Patriotic Health Campaign" and "Building Healthy Cities and Towns").

\subsection{Current situation of medical education and human resources for health}

Over the past years, China's health workforce has experienced a steady expansion of the total health workforce and significant changes in its composition. In 2015, the number of licensed physicians (and assistant physicians) was 2.2 per 1000 population, and that of registered nurses was 2.4 per 1000 population. However, there are critical challenges concerning the distribution of the Chinese health workforce between urban and rural areas, as well as severe shortages of the health workforce in some areas.

In 1998, China started an ambitious reform of the world's largest medical education system. From 1998 to 2012, the average growth rate of medical enrollees was $15 \%$, which was around 7.1-fold increase within 15 years [2]. China's medical education system ranks among the largest in the world regarding the number of enrollees, undergraduate and graduate students, and the total amount of trained health professionals, which plays a critical role in solving the shortage of health workforce.

A series of key policies have been issued to solve the healthcare human resource crisis in China including the establishment of a general practitioner (GP) system, standardized resident training, and targeted medical education programs. However, the implementation of these policies varies radically from place to place. The impact of these policies should be monitored and evaluated systematically to provide further evidence for nationwide implementation.

\subsection{Healthcare legal system in China}

Since the 1980s, China has built a comprehensive healthcare legal system. This legal system has taken the national constitution as the principle, 12 healthcare laws as the core, with dozens of health administrative regulations as the main body and more than 100 departmental regulations and numerous norms as the 
bases. The present healthcare legal system is a cluster of single laws. Usually, a single law is successively introduced to solve a specific problem related to medicine and healthcare. These healthcare laws and regulations adopt the leading ideas by governing, regulating, and restraining, which have played a critical role in the development of medicine and health in China in recent years.

\section{Challenges faced by the national health education, medical education, and healthcare legal system}

\subsection{Problems and challenges faced by the national health education system}

3.1.1 Health promotion legislation and coordination mechanisms are absent

Many developed countries guarantee health education implementation by law and specify the responsibility of allocation of all the departments involved. In the 1970s, the United States of America passed the National Health Planning and Resources Development Act and the National Consumer Health Information and Health Promotion Act of 1976. In 2002, Japan promulgated the Health Promotion Act, and the Labor Safety and Health Act focused on workplaces. Nowadays, there is an absence of a health promotion act in China and the duties of departments are unclear; hence our country still lags behind the advanced global level.

\subsubsection{Health education in China lacks enforcement}

Many developed countries have laws to enforce health education in schools and workplaces and treat health education as essential content for citizen education. Taking the United Kingdom as an example, schools are required to ensure at least one-hour of health education each week, and students need to undergo a unified health education assessment. According to the Labor Safety and Health Act in Japan, enterprises with more than 50 employees must employ occupational health doctors offering health education and health consultation services in a planned way [3].

At present, health education policies in China lack enforcement. The basic public health services encompass health education that is financially guaranteed, but is mainly directed at elderly people in communities. It is difficult to enter schools, enterprises, and workplaces; therefore, health education cannot be extended to a broader range of the population. The actual coverage of health education is relatively low. Schools are a major battlefield for health education. In 2008, the Ministry of Education in China issued the Health Education Guiding Outlines targeting at primary and secondary schools but did not set mandatory credit requirements for health education. Usually, physical education teachers, head teachers, school doctors, and biology teachers in primary and secondary schools give lectures on health education. Schools lack full-time health education teachers [4]. Health education in workplaces is being piloted for a long time. Similarly, health education in hospitals is also poorly funded and lacks policy support.

3.1.3 Planning and implementation strategies for health education need to be improved, and the capacity of health professionals strengthened

At present, the binding force of health education laws and regulations is insufficient. The whole health education system lacks personnel, funding, monitoring, and evaluation, hindering the continuous effective assessment of health education and its promotion, as well as providing further evidence for policymaking and legislation.

The lack of investment in health education affects the implementation strategies. There is no clear-cut limit for health education among government funding on basic public health services, which can easily lead to the restriction of health education expenditure. The subsidy for the health literacy promotion project from the central government is less than 0.2 yuan per capita. Mass media's duties regarding health education are shifted to the market, resulting in considerable false information and people being misled.

The training system of health professionals and the capacity of health education need to be strengthened. In 2013, 81.4\% counties in China set up health education institutions, while the percentage was only $64.7 \%$ in the less developed western provinces. Half of the staff in health education institutions works part-time. Communities, schools, workplaces, and even hospitals lack professional staff with expertise in health education.

\subsection{Problems concerning medical education and healthcare human resources}

(1) Medical education quality has been affected while professional health training efficiency has decreased dramatically. The percentage of undergraduate enrollees kept descending while the percentage of junior college enrollees kept ascending. During the period of 1981-2012, the gap between the number of medical graduates and healthcare professionals was expanding, implying that more and more health workers graduated from college over this period were not absorbed into the health workforce [2].

(2) Problems of uneven geographical distribution of health workforce and imbalanced skill mix are also found in China. The gap between the western, central, and eastern regions has been expanding. The number of pediatricians, psychiatrists, technicians, nutritionists, and community nurses is certainly inadequate. There is also a significant shortage of geriatric medical professionals making it arduous to combat the challenges brought by an aging population. Medical training has also been criticized due to the emphasis on hospital practice, with little exposure of GPs, resulting in the lagging behind of GPs' quantity and quality.

(3) Some training programs for health professionals face several 
difficulties. Concerning training for GPs, there are problems such as slow training, brain drain, weak implementation and limited effectiveness of job-transfer training, and lack of GPs, among others. In terms of standardized resident training for physicians, the following issues are prominent: income of residents is relatively low; training bases are not enough; and allocation of medical graduates is unsuitable. Problems can also be found in the medical education program including less motivated students, greater graduate employment difficulties, and inadequately implemented standardized resident training [5].

\subsection{Problems within the healthcare legal system}

(1) The present healthcare legal system needs a timely update. Along with the rapid economic and social development in China, the health industry also experienced ideological change, technology innovation, and organizational changes, resulting in the incompatibility of the healthcare legal system with a rapidly developed health system. Therefore, we need to improve the internal consistency and external adaption of the healthcare legal system enforcing dynamic and timely management.

(2) Laws should be enacted to guarantee the success of the healthcare development experience. China's health system has accomplished great achievements in improving healthcare over the past 30 years. For example, the new rural cooperative medical program has rapidly expanded the coverage of financial protection for rural residents. Such experiences should be verified, supported, and protected by national laws.

(3) "Parent Law" is absent from the current healthcare legal system. Having access to healthcare is a basic human right; therefore, the state has the obligation to protect the basic rights of its citizens. A set of fundamental principles or established precedents such as the "Parent Law" is needed in accordance with other legislations in the healthcare legal system, normalizing it in terms of principles, purposes, and concrete norms. Thus, when we make the "Parent Law" for the healthcare field, we should specify the rights and obligations of both the government and the related subjects, as well as citizens' health-related rights. The law will play a crucial role in universal health coverage, regulating and ensuring the fair and efficient operation of public institutions and social structures.

\section{Policy recommendations to improve national health education, medical education, and healthcare legal system}

\subsection{Recommendations for establishing a national health education system}

In establishing a national health education system, the following factors are important for its success: health education should be centered at schools and workplaces, regard the media as an important resource to educate the population, and utilize other social forces to supplement these efforts. The national health education system should provide services ranging from prevention, treatment, and rehabilitation, covering both healthy and nonhealthy people and encompassing every stage of life.

Regarding the implementation strategy, we need to redesign the "National Patriotic Health Campaign Committee" into a "National Council for Health Promotion." We must coordinate various departments, clarify the responsibilities of central and local governments each, and issue and carry out relevant policies to ensure a reasonable planning and effective implementation of health education. We should develop a public health education system that covers the entire population and works effectively, strengthening the school health education in particular. We should also improve the competencies of health education professionals, support the grassroot networks, and increase the investment in health education.

\subsection{Recommendations for improving medical education and} healthcare human resources

Efforts to scale up health professionals' training must not only show an increase in quantity but also address issues of quality to address population health needs. Training programs should be scaled up to produce high-level service delivery teams for the western regions. A targeted medical education program should be supported and promoted to ensure that sufficiently qualified health professionals are available and prepared to deliver effective services in primary healthcare institutions and rural areas.

Well-designed human resource policies are required, and distribution, compensation, and job subsidies should be implemented in primary healthcare institutions to assure a carefully balanced mix of clinicians, community health workers, and health managers. It is clearly urgent and essential to make primary healthcare suited to the health needs of the populations and guarantee equitable access to health services.

\subsection{Recommendations for healthcare legal system}

Promoting the introduction of "Parent Law" in medicine and healthcare is urgent. The relationship between the "Single Law" and the "Parent Law" should be balanced, so that they maintain their respective roles. The contents of "Parent Law" in medicine and healthcare includes but is not limited to: basic principles of protecting civil rights of life and health, medical services, public health services, health promotion, health-related products, health financing, supervision, and administration, among others.

Meanwhile, we need to carefully examine and analyze present medical and healthcare laws and regulations to propose legal reservation, amendments, or abrogation suggestions, and realize the dynamic management of the healthcare legal system in the development of the national health education system. 


\section{References}

[1] Community Health Association of China. Study on implementation and needs assessment of health education services in primary health care facilities [R]. Beijing: Community Health Association of China, 2013. Chinese.

[2] Hou J, Michaud C, Li Z, et al. Transformation of the education of health professionals in China: Progress and challenges [J]. Lancet, 2014 (384): 819-827.

[3] Qian J. Comparison of school health education of China, Japan and the United States at basic education stage [J]. Journal of Xi'an Physical Education University, 2008(2): 120-123. Chinese.

[4] Ma J. Opportunities and challenges in current school health [J]. Chinese Journal of School Health, 2012(1): 1-4. Chinese.

[5] Hu D, Chen C K, Zhang C, et al. Policy implementation of targeted admission medical education program in rural areas [J]. Chinese Journal of Health Policy, 2016, 9(9): 60-64. Chinese.

[6] Tang C. Analysis of the development status of health law education in America [J]. Medicine and Society, 2013, 26(2): 84-86. Chinese. 